\title{
How the surrounding environment affects the biosensing performance of resonant nanopillars arrays: Under dry conditions or immersed in fluid
}

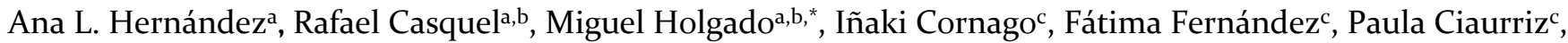 \\ Francisco J. Sanza d, Beatriz Santamaría ${ }^{a}$, María V. Maigler ${ }^{\mathrm{a}, \mathrm{c}}$, Sergio Quintero ${ }^{\mathrm{a}}$, María Fe Laguna ${ }^{\mathrm{a}, \mathrm{b}}$
}

\footnotetext{
a Centre for Biomedical Technology, Universidad Politécnica de Madrid, Campus de Montegancedo, Pozuelo de Alarcón, 28223, Madrid, Spain

b Department of Applied Physics, Escuela Superior de Ingenieros Industriales, Universidad Politécnica de Madrid, C/José Gutiérrez Abascal, 2, 28006, Madrid,

Spain

${ }^{c}$ Cemitec, ADItech, Polígono Mocholí, Plaza Cein, 4, 31110, Noain, Spain

d Bio Optical Detection, Centro de empresas de la Universidad Politécnica de Madrid, Pozuelo de Alarcón, 28223, Madrid, Spain
}

\section{A B S T R A C T}

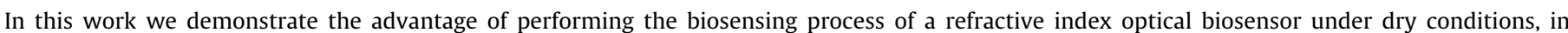

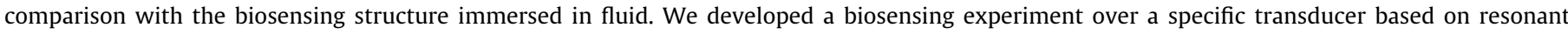

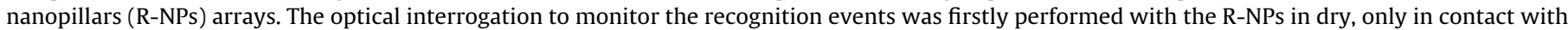

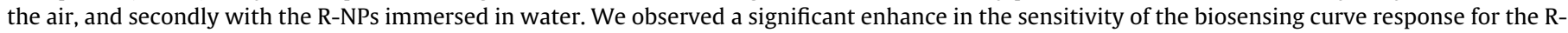

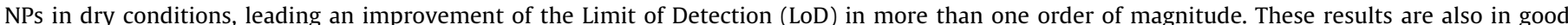

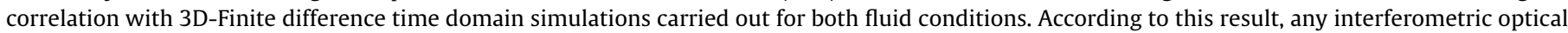

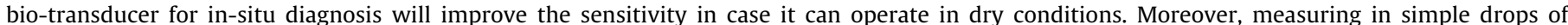
biological samples, in dry conditions, will be a relevant issue for Point of Care Devices.

\section{Keywords:}

Biosensing enhance between dry/wet conditions

Optical biosensor

Resonant nanopillars

In-situ diagnosis

Vertical optical interrogation

Label-free

\section{Introduction}

High sensitivity optical detection methods are a continuous matter of study, in which scientific community is working persistently to achieve the development of novel biosensing systems with improved performance [1]. This work is specially focused on the field of micro and nano technology, where many optical integrated sensors and biosensors [2,3] have proven to be effective and strong candidates to become the technology on which the diagnosis systems lab-on-a-chip [4] and Point of Care detection devices (PoC) must be based [5-7].

Presumably the best known label-free optical biosensor is the SPR (Surface Plasmon Resonator) [8], which is based on the refrac-

* Corresponding author at: Centre for Biomedical Technology, Universidad Politécnica de Madrid, Campus de Montegancedo, Pozuelo de Alarcón, 28223, Madrid, Spain.

E-mail address: m.holgado@upm.es (M.F. Laguna). tive index change (RI) and is being widely used commercially. Moreover, last trends in research are addressing miniaturization and portability [9]. In addition, optical biosensors based on interferometric transducers can measure biological moieties by measuring the change in refractive index (RI) caused by the bioconjugates interaction [10]. Some examples of interferometric transducers are Mach Zehnder [11], BICELLs [12], Young interferometer [13] or photonic crystals [14], among others. Also optical waveguides and ring resonators are used in biosensors based on optical interferometry $[15,16]$.

Interferometric transducers based on R-NPs are an efficient technology for emerging diagnosis systems. The optical properties of the materials of which the R-NPs are formed and the specific distribution of these materials along the pillars, produce a sharp resonance mode formation when R-NPs are vertically interrogated, due to the central cavity between the Bragg Reflectors forming the R-NPs.

In previous works we demonstrated the performance of R-NPs made of $\mathrm{Si}_{3} \mathrm{~N}_{4} / \mathrm{SiO}_{2}$ [17] and their feasibility to be used as biochem- 
ical sensors [18,19]. For it, we immersed the pillars in fluids with different refractive index (RI), and we interrogated them vertically from the backside. R-NPs showed high throughput in detecting small changes in the RI of the media in which they were immersed. The biological events produce shifts in resonance modes suitable to be monitored along the immobilization of bioreceptors or during the specific recognition of target biomolecules. Thereby, in a different former study we confirmed the possibility of binding biomolecules to R-NPs surface and therefore their feasibility to work as multiplexed label-free optical biosensors, where the response of identical R-NPs arrays transducers exhibited a good reproducibility [20]. In that work, a standard immunoassay was developed over the pillars for the biosensing demonstration.

In the present work we study the comparison of the biosensing performance under dry conditions or immersed in fluid of a transducer based on RNPs arrays. We demonstrate through a biosensing experiment, the increased performance of R-NPs when they are vertically interrogated in dry conditions. The obtained results can be extended to other interferometric optical biosensors reported in the literature.

\section{Materials and methods}

In this work we compare the biosensing response of the same R-NPs arrays by using two different characterization modes. One of them is with the R-NPs immersed in the dilution sample; in this case water, but depending of the assay could also be Phosphate Buffer Solution (PBS) or other biological sample (Fig. 1-A). The other is with the pillars in dry conditions (Fig. 1-B). The drying process effect was previously reported as an efficient method to accurately carry out immunoassays where biosensing signals fit properly with the theoretical calculations $[7,12,20,21]$.

We developed an IgG/anti-IgG standard immunoassay purchased from Sigma Aldrich (I4231/R3756) for the comparison of the biosensing performance of the two different interrogation procedures. All the experiments were carried out under controlled room temperature at $20^{\circ} \mathrm{C}$ plus/minus $2^{\circ} \mathrm{C}$. Humidity was also stable during the experiments.

The chip developed is composed by eight different R-NPs arrays of $1 \times 1 \mathrm{~mm}^{2}$. We call BICELLs (Biophotonic Sensing Cells) to these arrays when they are biofunctionalized with the bio-receptors [21]. The chip's layout has distributed 2 rows and 4 columns of BICELLs over a quartz substrate. This distribution of BICELLs provides multiplexing features to the sensor chip. R-NPs are formed by 10 pairs of Bragg Reflectors $\left(\mathrm{Si}_{3} \mathrm{~N}_{4} / \mathrm{SiO}_{2}\right)$ with a central cavity of $200 \mathrm{~nm}$ of $\mathrm{SiO}_{2}$. The lattice parameter among the pillars forming the array is $500 \mathrm{~nm}$ and the pillars diameter is around $200 \mathrm{~nm}$. The fabrication of the R-NPs involves several steps, from thin film layer deposition, laser interference lithography, an intermediate lift-off process and a final etching of the pillars; this fabrication process has been reported in detail elsewhere [17].

The chip was located in a holder-fabricated ad-hoc with the pillars facing down, as described in previous works [20]. Surface modification, biofunctionalization and biological recognition process for the immunoassay purpose affected to all the R-NPs arrays from the sample chip. However, according to the aforementioned previous work, we assumed that the biosensing response would be similar for every BICELLs, thus in this work the presented comparison data correspond to the characterization of one single BICELL (R-NPs array) from the multiplexed chip. After every sensing step, the arrays were independently, vertically interrogated with a bundle of fiber with a beam spot size of $500 \mu \mathrm{m}$ diameter, with a spectrometer (with a resolution 0 of $0.1 \mathrm{~nm}$ ), covering the visible range in which the resonance mode can be observed. (Fig. 1-C). Every step of the immunoassay was characterized within the two comparing methodologies: on one side, by immersing the pillars in water and on the other hand, with the pillars in dry conditions.

For the immunoassay over the R-NPs, they were firstly biofunctionalized with the IgG. For that reason, we developed a silanization protocol based on 3-aminopropyl trimethoxysilane (APTMS), and glutaraldehyde (GA) as linker to the amine groups of the antibody, which is further described in our previous work [20]. The immobilization of the IgG over R-NPs was carried out through the incubation of a drop of $50 \mu \mathrm{g} / \mathrm{mL}$ of IgG, over night and in humid conditions in order to avoid the evaporation of the sample drop. Subsequently the chip with the R-NPs was rinsed with PBS-tween and water and properly dried with clean air. Then the chip was placed on the empty holder (with the R-NPs facing down) for the interrogation of the R-NPs in dry conditions and later on, the holder was filled with water for the interrogation of the R-NPs in humid conditions. Secondly, this process of incubation, rinsing, drying and both methods of interrogation, (in dry conditions and with the R-NPs immersed in water), was repeated for the recognition of increasing concentrations of anti-IgG with no regeneration phase between them. The incubation time of the increasing concentrations of anti-IgG was $30 \mathrm{~min}$ (Fig. 2).

Finally, we have performed theoretical calculations for the RNPs spectra using the commercial software RSoft [22], and in particular its feature for Finite Difference Time Domain calculations Fullwave [23]. We have obtained reflectivity referred to silicon of the photonic structure for both dry and immersed in water conditions, considering a variable thickness biofilm layer with a refractive index of 1.41 surrounding the pillars, with a thickness ranging from 0 to $20 \mathrm{~nm}$. For the calculations we have considered three-dimensional space domain, with a grid resolution varying from $20 \mathrm{~nm}$ for the bulk pillars, to $5 \mathrm{~nm}$ for the biofilm layer.

\section{Results and discussion}

We vertically interrogated the BICELLs without any previous treatment in order to have a reference signal of the spectral mode for both characterization procedures: with pillars immersed in water and with pillars in contact with air (Fig. 3).

In Fig. 3, the pink line represents the reflectance signal as a function of the wavelength when the R-NPs array is untreated and in dry conditions. The Blue spectrum corresponds to the untreated RNPs array immersed in water. It can be observed a smaller intensity of the transducing signal in liquid infiltrated R-NPs, which barely reaches 1.5 relative units of the reflectance referred to silicon. Besides, the resonance mode is centered at $582 \mathrm{~nm}$, in comparison, when the pillars are in dry conditions; it is centered at $485 \mathrm{~nm}$.

Two other parameters may be mention although, in this case they are not decisive to determine biosensor performance: the signal-to-noise ratio (SNR) and the width of the resonance dip, expressed as the Full Wave at Half Maximum (FWHM). Both parameters provide information about the biosensor yield [24]. For the same transducer, a higher SNR ratio and a smaller FWHM are observed for the R-NPs in dry conditions.

The relative shift of the resonant deep during the silanization process and during biofunctionalization (IgG immobilization onto the sensing surface), for both measuring typologies, is represented in Fig. 4. The pink bars show the shift of the R-NPs resonance position after every biofunctionalization step, when the R-NPs are dry. The shift is around $2.2 \mathrm{~nm}$ after APTMS silanization and $6.14 \mathrm{~nm}$ for the IgG immobilization over the silane layer. For the same R-NPs immersed in water (blue bars) the shift is $0.4 \mathrm{~nm}$ for the APTMS and $1.23 \mathrm{~nm}$ for the IgG attachment. These results do not imply that the amount of antibody attached on the surface of the pillars is lower for pillars immersed, because it is the same R-NPs sample. However, they reveal that the changes occurring over biosensing structures, 

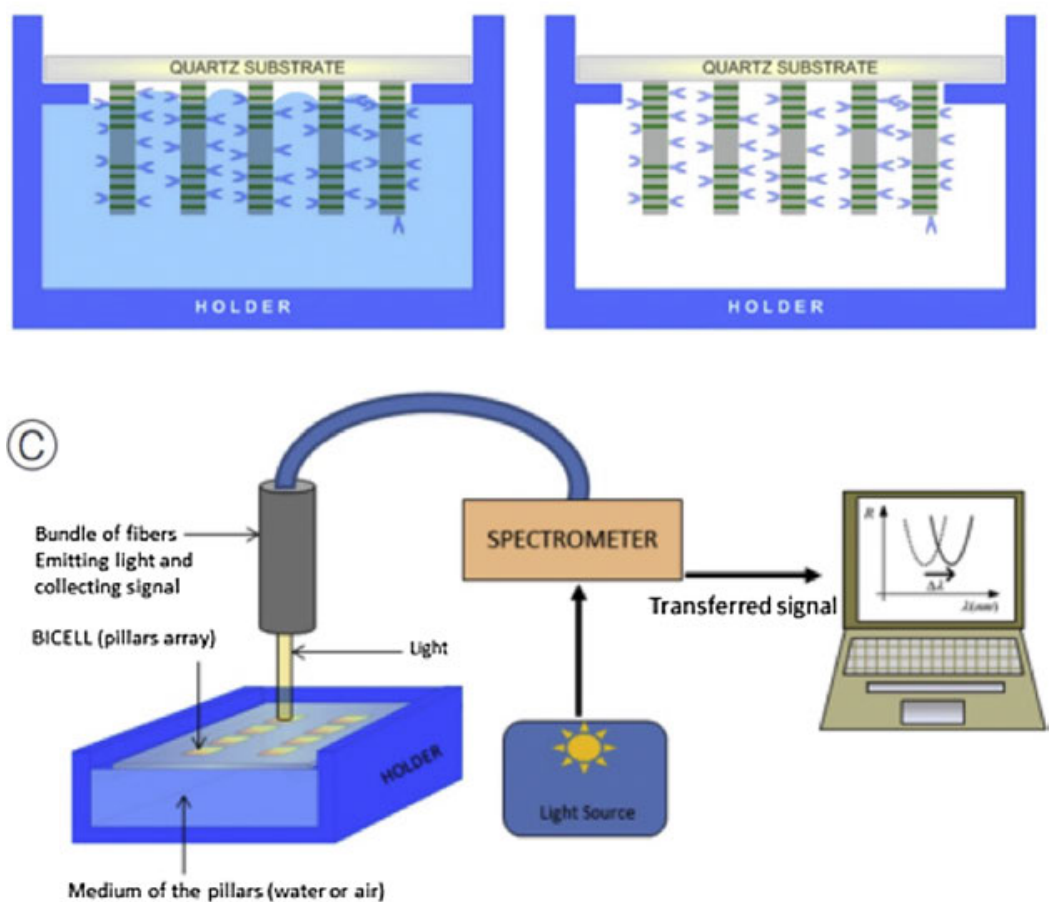

Fig. 1. A. Scheme of pillars immersed in water. B. Scheme of pillars in dry conditions. C. Layout of the measurement system.

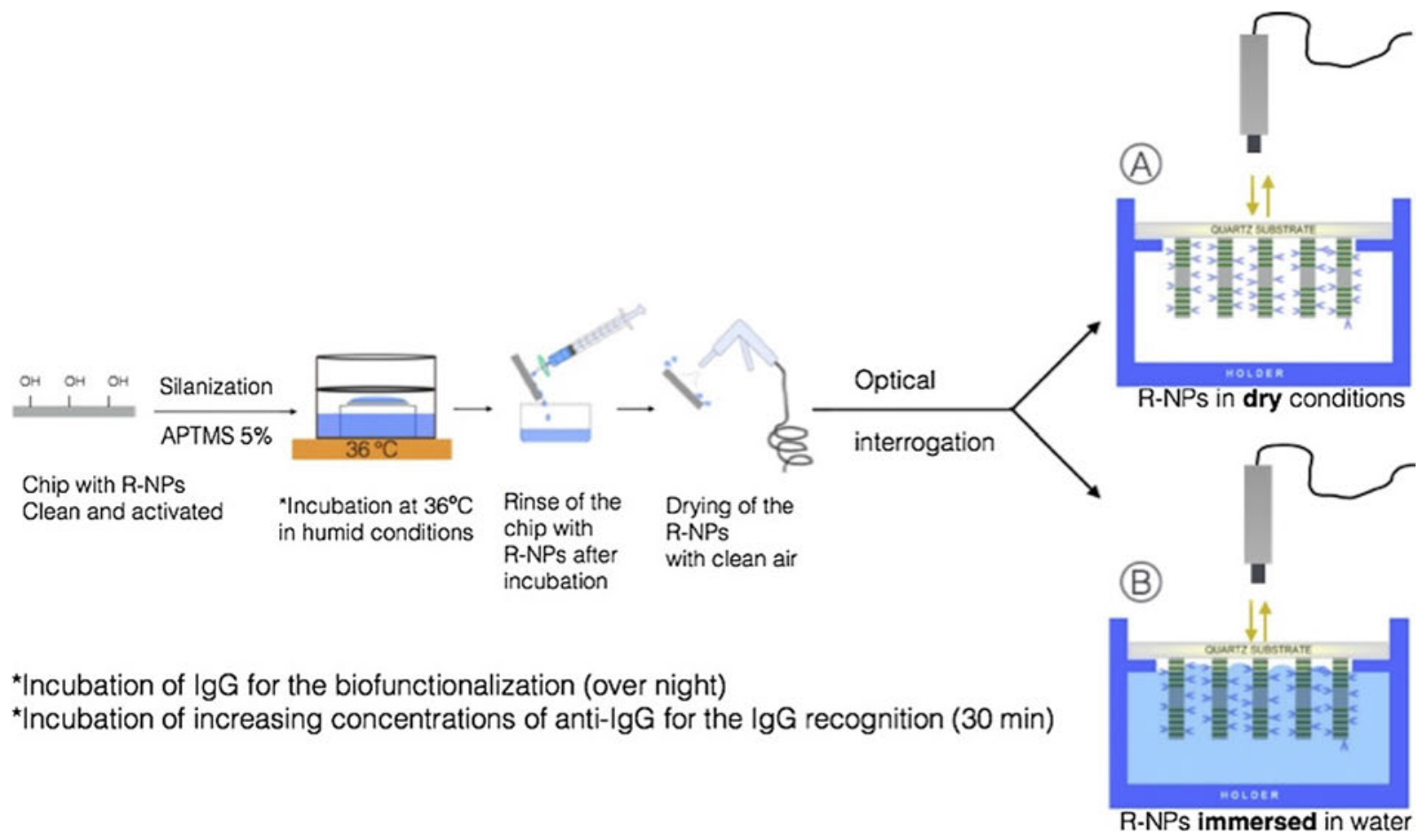

Fig. 2. Process carried out for the interrogation of the immunoassay steps with both methods, (A. with R-NPs dry and B. with R-NPs immersed).

are more easily observed when R-NPs are not immersed. The shift corresponding to the IgG immobilization is approximately 3 times the shift of the silane layer formation.

As summary, we can state that higher wavelength displacement results in greater sensitivity (m). Also, due to the lower FWHM and higher SNR, the uncertainty $(u)$ on the calculation of the minimum position of the resonance, is smaller for R-NPs interrogated in dry conditions.

Thus, due to these figures, we expect to achieve a better performance in terms of limit of detection (LoD) under dry conditions. In order to demonstrate and check these abovementioned assumptions, we carried out a recognition experiment to obtain the biosensing curve response (Fig. 5-A). For this recognition phase, 


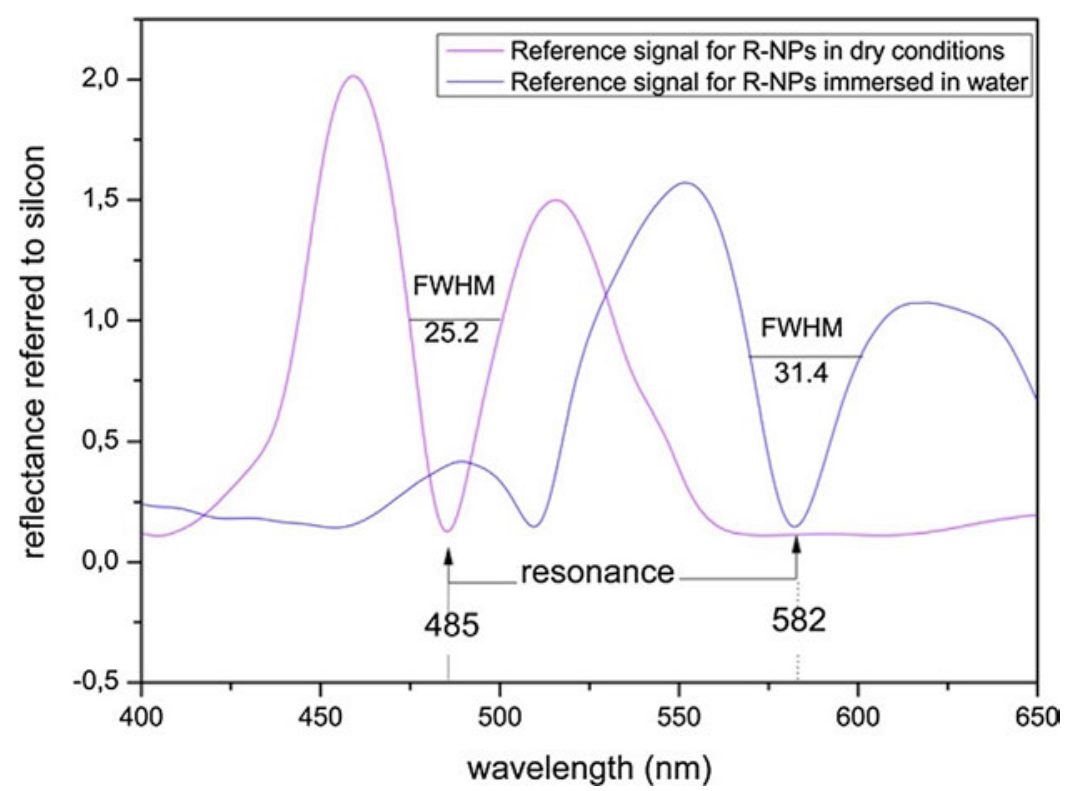

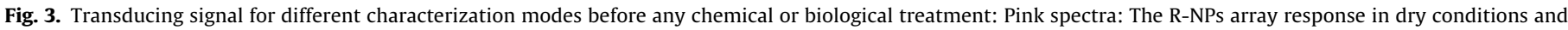

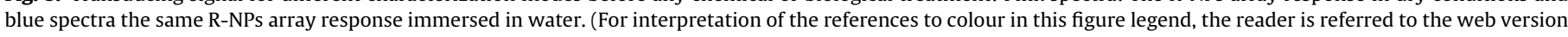
of this article.)

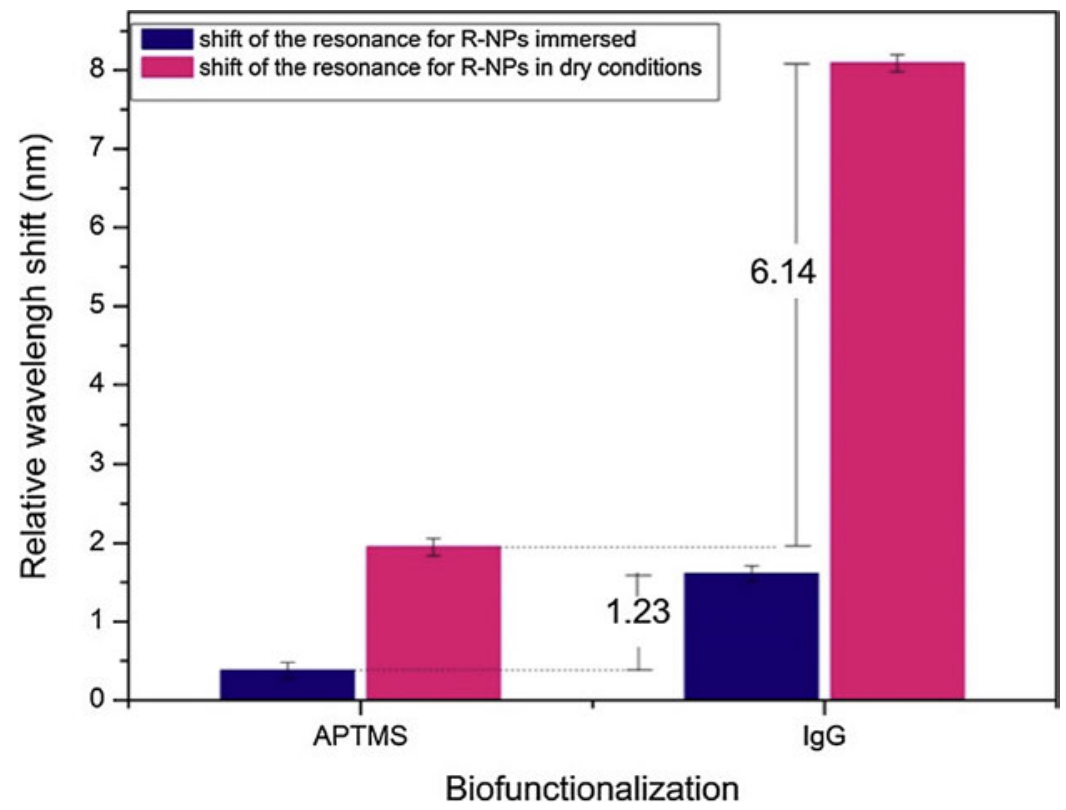

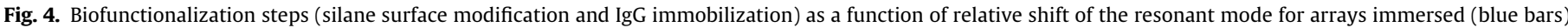
and not immersed (pink bars). (For interpretation of the references to colour in this figure legend, the reader is referred to the web version of this article.)

different concentrations of anti-IgG were incubated in the R-NPs BICELLs. The point in 0 represents the stable biolayer of IgG and the subsequent points correspond to the relative shift for the increasing concentrations of anti-IgG $(2.5,5,10,15,20$ and $50 \mu \mathrm{g} / \mathrm{mL})$. Fig. 5-B and C corresponds to the transducing signal of the R-NPs for the IgG is immobilization and for the recognition of anti-IgG at a concentration of $50 \mu \mathrm{g} / \mathrm{mL}$, assuming that surface is saturated at that concentration. It can be seen the higher resonant mode shift for interrogation of R-NPs in dry conditions.

The relative shift of the highest concentration of anti-IgG recognition for the dry pillars, is $4.36 \mathrm{~nm}$ and for the immersed pillars is $0.68 \mathrm{~nm}$. Knowing the IgG and anti-IgG both biomolecules have the same size (around $12 \mathrm{~nm}$ ), it would be expected a similar shift for the recognition of the anti-IgG, than for the immobilization of IgG
(6.14 and 1.23 for R-NPs dry and in humid condition respectively) if the yield of the recognition was $100 \%$. Therefore we assume that probably the orientation of the IgG was not the ideal, and consequently there were some fragment antigen areas (Fab), which were not available for the recognition process.

Fig. 6 shows the reflectivity spectra calculated using 3D-FDTD algorithm, without considering an attached biofilm layer. The effect of adding this layer is a shift in the resonant mode; in the case of dry conditions this mode is centered around $484 \mathrm{~nm}$, whereas for water conditions the dip is around $570 \mathrm{~nm}$ (Fig. 6-A). We have calculated the shift of the resonance mode when adding a biofilm layer of 5, 10, 15 and $20 \mathrm{~nm}$ (Fig. 6-B), for both fluid an dry conditions. The total shift, considering $20 \mathrm{~nm}$ of biofilm is of $2.84 \mathrm{~nm}$ (immersed in water) and $16.32 \mathrm{~nm}$ (dry conditions); more than 5 


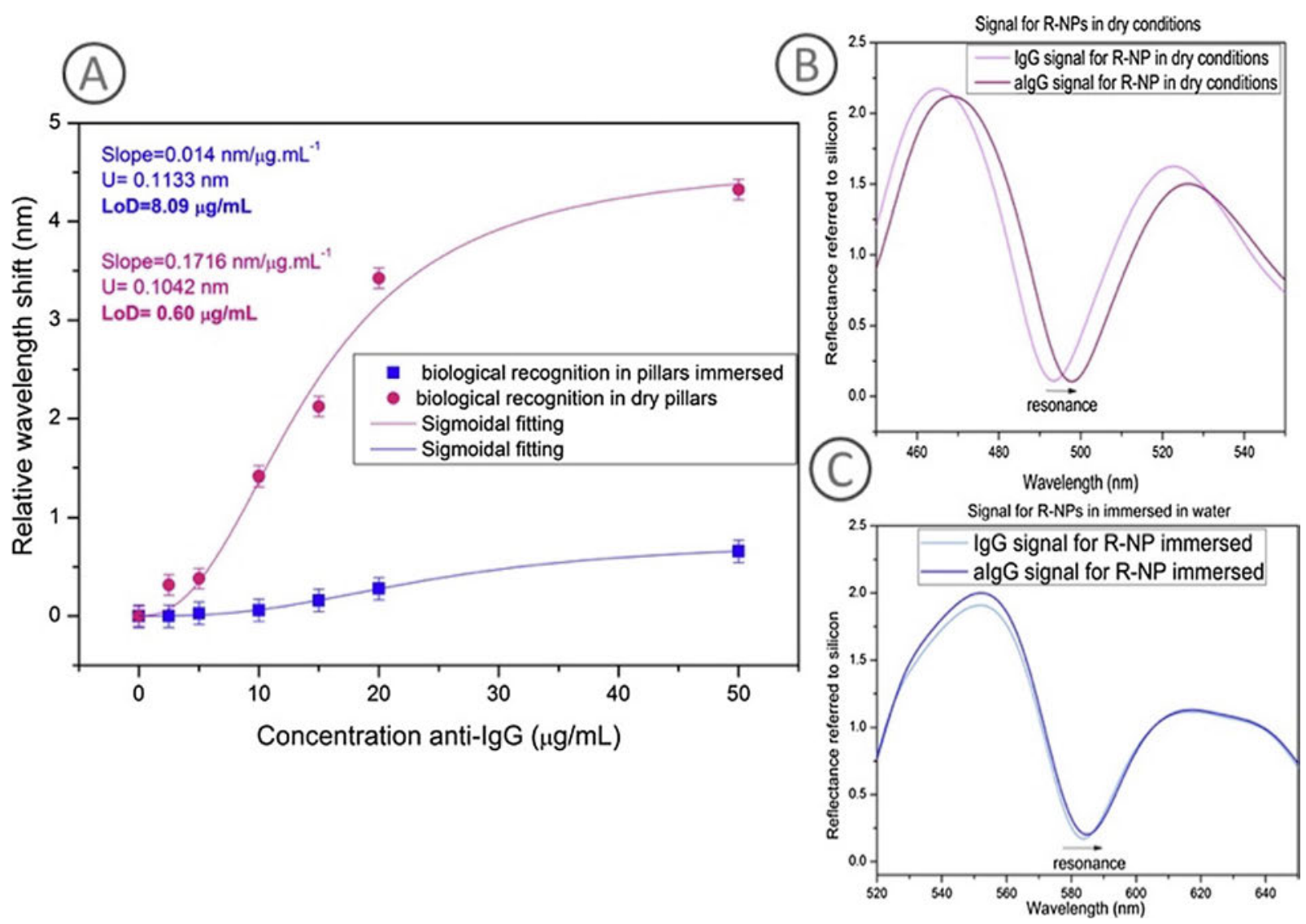

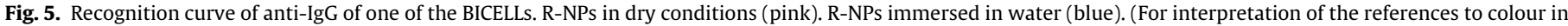
this figure legend, the reader is referred to the web version of this article.)
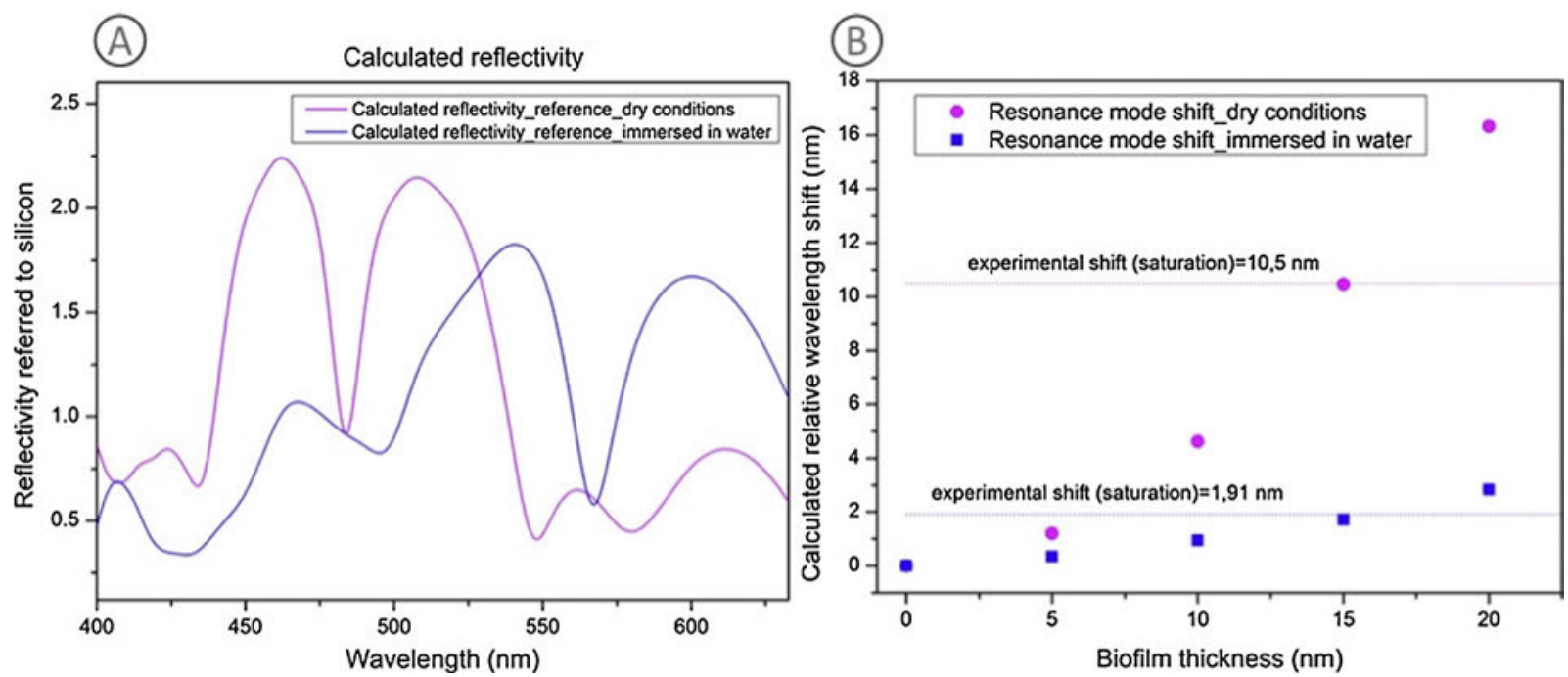

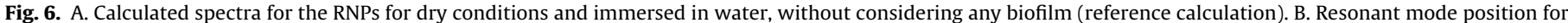
calculated spectra as a function of biofilm thickness considered, from 0 to $20 \mathrm{~nm}$, for both fluid conditions.

times (5.75) higher for dry conditions. The maximum shift reached experimentally is of $2.31 \mathrm{~nm}$ (water) and $12.7 \mathrm{~nm}$ (dry), considering the biofunctionalization of IgG plus the anti-IgG recognition shift; 5.5 times higher for dry conditions in this case. If we compare experimental and theoretical data, we observe that the saturation shift for both conditions seems to correspond in the two cases with a biofilm thickness around $17-18 \mathrm{~nm}$ (Fig. 7-B). This is in accordance with the relatively low shift for the biorecognition process, and thus a bit lower than expected (a biofilm layer of $20-25 \mathrm{~nm}$ was expected for this calculations).

After every recognition step, 7 repeated measurements were taken in order to obtain the standard deviation of the resonance position. For the standard uncertainty $(u)$ calculation, it was considered the resolution of the measurement system $(R)$, the number of repeated measurements ( $\mathrm{n}$ ) and its standard deviation (s) as expressed in Eq. (1) [25]. This Eq. (1) is the law of propagation of uncertainty, where other factors, such as the temperature are 
Table 1

Quality parameters of both interrogation techniques.

\begin{tabular}{|c|c|c|c|c|}
\hline & FWHM nm & Uncertainty (U) nm & Sensitivity $(\mathrm{m}) \mathrm{nm} /\left(\mu \mathrm{g} \mathrm{mL}^{-1}\right)$ & Limit of Detection (LoD) $\mu \mathrm{g} / \mathrm{mL}$ \\
\hline R-NPs immersed in water & 31.41 & 0.11 & 0.01 & 8.08 \\
\hline R-NPs in dry conditions. & 25.20 & 0.10 & 0.17 & 0.60 \\
\hline
\end{tabular}

considered negligible. The expanded uncertainty (U) was obtained with a coverage factor of 3 (Eq. (2)).

$u^{2}=\frac{R^{2}}{12}+\frac{s^{2}}{n}$

$U=3 \cdot u$

For a further comparison of the different techniques we calculated the LoD of the sensor under the two media conditions, air and water.

$L o D=\frac{U}{m}$

Where $m$ is the sensitivity, and is calculated as the slope of the linear range between the first point of recognition and the saturation concentration point. The aforementioned quality parameters comparing both measuring techniques are summarized in Table 1.

As it could be inferred from the resonant modes in Fig. 2, the standard uncertainty for the minimums identification, is higher when R-NPs are measured in wet conditions, due to a lower SNR and a higher FWHM. Therefore the expanded uncertainty $U$ is also higher for the immersed R-NPs.

Considering $20 \mu \mathrm{g} / \mathrm{mL}$ as the saturation point we observed that the sensitivity (m), when the R-NPs are dry, is one order of magnitude higher regarding wet pillars $\left(0.014\right.$ and $0.1716 \mathrm{~nm} / \mu \mathrm{g} \mathrm{mL} \mathrm{m}^{-1}$ respectively). Thus, sensitivity, which is the resonance shift as a function of the analyte concentration, has a greater influence on the LoD determining compared with the uncertainty, which is very similar for both cases of study. In accordance with uncertainty and sensitivity values, the LoD, expressed in Eq. (3), is improved 13.2 times if the experiment is performed with the pillars in dry conditions.

This improvement is mainly due to the higher shift of the resonance mode induced by the higher Refractive Index Contrast $(\Delta \mathrm{n})$ between R-NPs and air. The $\Delta \mathrm{n}$ between R-NPs and water is lower than the $\Delta \mathrm{n}$ existing between R-NPs and air. In wet conditions, the index of the surrounding media is 1.33 , and 1.41 for the biolayer, whereas in dry conditions the index of the surrounding media is 1 . This higher contrast results in higher values of the resonance shift, as seen experimentally.

\section{Conclusions}

In this work we evidence the result of measuring the same sample, with the same measuring system but in two different media: water and air. There have been demonstrated the benefits of measuring the biosensing response of R-NPs in dry conditions, where an enhancement in the limit of detection, in more than one order of magnitude is reached. Theoretical results are also in good correlation with experimental data. Moreover, the comparison results of this scientific report could be applied to other label-free optical transducers reported in the literature.

Finally, the R-NPs are susceptible to be fabricated at industrial level; moreover, their better performance in dry conditions makes them potentially ideal optical transducers to be implemented in Point of Care devices for in-situ diagnosis (portable and fast diagnosis systems), avoiding complex fluidic systems which are heavier, harder to handle and probably more expensive. This also could be applied to other interferometric transducers.

\section{Acknowledgments}

This project is supported by the funding agencies: European Commission, Project Enviguard (FP7-OCEAN-2013-614057) and Spanish Ministry “Ministerio de Economía y Competitividad”, under the projects PLATON (TEC2012-31145) and ATAPOC (Ref.: RTC2015-3273-1). Authors also acknowledgments to BIOD for the support on the holder fabrication and engineers Martin Domingo and Manuel Vicente for their contributions on the mechanical parts of the readout system.

\section{References}

[1] A.B. González-Guerrero, J. Maldonado, J.S. Dante, S.D. Grajales, L.M. Lechuga, Direct and label-free detection of the human growth hormone in urine by an ultrasensitive bimodal waveguide biosensor, J. Biofoton. 10 (2016) 61-67.

[2] X. Fan, I.M. White, S.I. Shopova, H. Zhu, J.D. Suter, Y. Sun, Sensitive optical biosensors for unlabeled targets: a review, Anal. Chim. Acta 620 (2008) 8-26.

[3] F.S. Ligler, Perspective on optical biosensors and integrated, Sens. Syst. Anal. Chem 81 (2009) 519-526.

[4] M.I. Mohammed, M.P.Y. Desmulliez, Lab-on-a-chip based immunosensor principles and technologies for the detection of cardiac biomarkers: a review, Lab Chip 11 (2011) 569-595.

[5] A. Romeo, T.S. Leung, S. Sanchez, Smart biosensors for multiplexed and fully integrated point-of-care diagnostics, Lab Chip 16 (2016) 1957-1961.

[6] C.D. Chin, V. Linder, S.K. Sia, Commercialization of microfluidic point-of-care diagnostic devices, Lab Chip 12 (2012) 2118-2134.

[7] M. Holgado, M.V. Maigler, B. Santamaría, A.L. Hernandez, A. Lavín, M.F. Laguna, F.J. Sanza, D. Granados, R. Casquel, J. Portilla, T. Riesgo, Towards reliable optical label-free Point-of-Care (PoC) biosensing devices, Sens. Actuators B- Chem. 236 (2016) 765-772.

[8] J. Homola, Surface plasmon resonance sensors for detection of chemical and biological species, Chem. Rev. 108 (2012) 462-493.

[9] N. Wonhwi, M. Kang, D. Jang, S. Shin, Miniaturized surface plasmon resonance biosensor with vacuum-driven hydrodynamic focusing, Sens. Actuators BChem 254 (2018) 64-71.

[10] D.P. Campbell, Principles of Bacterial Detection: Biosensors, Recognition Receptors and Microsystems, Springer, 2008, pp. 169-211.

[11] D. Yuan, Y. Dong, Y. Liu, T. Li, Mach-zehnder interferometer biochemical sensor based on silicon-on-insulator rib waveguide with large cross section, Sensors 15 (9) (2015) 21500-21517.

[12] M. Holgado, C.A. Barrios, F.J. Ortega, R. Sanza, R. Casquel, M.F. Laguna, et al., Label-free biosensing by means of periodic lattices of high aspect ratio SU-8 nano-pillars, Biosens. Bioelectron. 25 (12) (2010) 2553.

[13] A. Brandenbourg, R. Henninger, Integrated optical young interferometer, Appl. Opt. 33 (25) (1994) 5941-5947

[14] E. Chow, A. Grot, L.W. Mirkarimi, M. Sigalas, G. Girolami, Ultracompact biochemical sensor built with two-dimensional photonic crystal microcavity, Opt. Lett. 29 (10) (2004) 1093-1095.

[15] C.F. Carlborg, K.B. Gylfason, A. Kazmierczak, F. Dortu, M.J. Banuls, A. Maquieira Catala, A packaged optical slot-waveguide ring resonator sensor array for multiplex label-free assays in labs-on-chips, Lab Chip 10 (2010) 281-290.

[16] Y. Sun, X. Fan, Optical ring resonators for biochemical and chemical sensing, Anal. Bioanal. Chem. 399 (2011) 205.

[17] I. Cornago, A.L. Hernández, R. Casquel, M. Holgado, M.F. Laguna, F.J. Sanza, J. Bravo, Bulk sensing performance comparison between silicon dioxide and resonant high aspect ratio nanopillars arrays fabricated by means of interference lithography, Opt. Mater. Express 6 (2016) 2264-2272.

[18] A.L. Hernández, R. Casquel, M. Holgado, I. Cornago, F.J. Sanza, B. Santamaría M. Maigler, F. Fernández, A. Lavín, M.F. Laguna, Arrays of resonant nanopillars for biochemical sensing, Opt. Lett. 40 (2015) 2370.

[19] F. Fernandez, P. Ciaurriz, I. Cornago, R. Casquel, A.L. Hernández, F.J. Sanza, M.F. Laguna, M. Holgado, Optical sensor based on periodic array of resonant nanopillars for real time monitoring, Sens. Actuators B- Chem. 244 (2017) 323-326.

[20] A.L. Hernández, R. Casquel, M. Holgado, I. Cornago, F. Fernández, P. Ciaurriz, F.J. Sanza, B. Santamaría, M. Maigler, M.F. Laguna, Resonant nanopillars arrays for label-free biosensing, Opt. Lett. 41 (2016) 5430-5433.

[21] M.F. Laguna, F.J. Sanza, J. Soria, M. Jara, Á. Lavín, R. Casquel, A. López, T. Suarez, M. Holgado, Label-free biosensing by means of BICELLs for dry eye, Sens. Actuators B- Chem. 203 (2014) 209-212.

[22] https://optics.synopsys.com/rsoft/. 
[23] R. Scarmozzino, A. Gopinath, R. Pregla, S. Helfert, Numerical techniques for modeling guided-wave photonic devices, IEEE J. Sel. Top. Quantum Electron 6 (2000) (2000) 150-162.

[24] J. de Vicente, M. Holgado, A. Lavín, F.J. Sanza, R. Casquel, M.F. Laguna, Uncertainty in optical bio-sensors due to the spectral displacement of the interferences modes of the transducing signal, OPA 47 (2014) 27-34.

[25] Guide to the Expression of Uncertainty in Measurement. https://www.bipm. org/en/publications/guides/gum.html.

\section{Biographies}

Ana L. Hernandez, MSc, degree in Environmental Sciences at Universidad Autónoma de Madrid. Postgraduate degree in "International Master of Inland Water Quality Assessment" taught jointly by the Mälardalen University and Universidad Autónoma de Madrid. Currently, she is PhD candidate developing her doctoral degree at the Center for Biomedical Technology (CTB-UPM) in the area of surface biofunctionalization and optical-label free biosensing.

Rafael Casquel received his PhD from Universidad Politécnica de Madrid (UPM) in 2012. Currently he works as Assistant Profesor at UPM, and is involved in the research in the field of optical biosensors within the Centre for Biomedical Technology, also in the UPM.

Miguel Holgado, I received my Bachelor's and Master's degree in Electrical Engineering from Technical University of Madrid (UPM) (1996), and Doctoral degree ( $\mathrm{Ph} . \mathrm{D}$ ) at the Institute of Material Science (ICMM) belonging to the Spanish National Research Council CSIC (2000). I am currently Deputy Vice- Rector for Innovation, group leader of the Optics, Photonics and Biophotonics at the Center for Biomedical Technology CTB-UPM, and associate professor at the Applied Physics and Material Engineering Department of Industrial Engineering School (ETSII-UPM).

Iñaki Cornago did his master degree in Materials and Fabrication Engineering and finished his PhD degree, focused on nanostructures fabrication processes, in 2015. He works at Cemitec since 2008, being involved in several projects related with biosensing and energy.

Fátima Fernández received her $\mathrm{PhD}$ in analytical chemistry from University of Barcelona (2011). Her research interests include nanobiotechnology, immunoassays and biosensors for development of novel diagnostic tools based on the use of bioreceptors.
Paula Ciaurriz graduated in Chemistry (2010) followed by a Master degree in Agrobiotechnology at the University of Navarra (2013). She is currently in the final steps of her PhD at Cemitec on the functionalization of nanoparticles and nanopatterned surfaces for immunosensing and signal enhancement applications.

Francisco J. Sanza, Francisco I received Ph.D. degree from Universidad Politécnica de Madrid (UPM) in 2015, IT Engineer degree in 2008 (Universidad de Navarra, Spain), a M.Sc. in Photonics in 2009 (Universidad Autónoma de Madrid) and a M. Eng. in Laser Technology in 2011 (UPM). He is currently technical director of Bio Optical Detection, a spin-off of the UPM and member of the Optics, Photonics and Biophotonics Group.

Beatriz Santamaría, I received my Bachelor's (2012) and Master's degree (2014). am currently PhD candidate at the Optics, Photonics and Biophotonics group at the Center for Biomedical Technology CTB-UPM in the area of micro-nano fabrication and surface bio-functionalization and assay measurements.

María V. Maigler, I received my Bachelor's (2013) and Master's degree (2015) in Electrical Engineering from Technical University of Madrid (UPM). I am currently $\mathrm{PhD}$ candidate at the Optics, Photonics an d Biophotonics group at the Center for Biomedical Technology CTB-UPM and Head of Unit of Electronic Unit at Bio Optica Detection, a spin-off of the UPM. I have led and participated in several European and National research projects.

Sergio A. Quintero, I received my Bachelor's (2015) in Electrical Engineering from Universidad Migule Hernandez) and Master's degree (2015) in Biomedical Engineering form Technical University of Madrid. I am currently PhD candidate at the Optics, Photonics an d Biophotonics group at the Center for Biomedical Technology CTB-UPM.

María Fe Laguna, received the Ph.D. degree in chemistry (1999) at the Polymer Institute form the Spanish National Research Council (CSIC), Madrid. She is deputy research group leader of the Optics, Photonics and Biophotonics group and professor at Applied Physics Department of the Technical University of Madrid. 